\title{
STATISTICAL MODELING OF SIGNAL AMPLITUDE FADING OF INDOOR RADIO PROPAGATION CHANNELS
}

\author{
Homayoun Nikookar* \\ Homayoun Hashemi** \\ * Dept. of Electrical Engineering, Amirkabir University of Technology, Hafez Street, Teheran, Iran \\ ** Dept. of Electrical Engineering, Sharif University of Technology, P. O. Box 11365-9363, Teheran, Iran
}

\begin{abstract}
Small-scale and large-scale variations of signal amplitude for wideband data collected at two office buildings have been investigated. The data base includes 12000 impulse response estimates which were obtained by inverse Fourier transforming of the channel's frequency response profiles. Extensive curve fitting for the distribution of individual multipath components' amplitudes using elaborate tests based on the KolmogorovSmirnov and Wilcoxon procedures shows that amplitude fading is lognormal for both local and global data. For the local data, the lognormal, Nakagami, and Rayleigh distributions passed the Kolmogorov-Smirnov test with a $90 \%$ confidence level for $76 \%$, $36 \%$, and $13 \%$ of cases, respectively. For global data the Wilcoxon test showed that with $99 \%$ confidence lognormal is better than Nakagami, and with $94 \%$ confidence Nakagami fits the data better than Rayleigh.
\end{abstract}

After establishing the lognormality of signal amplitude fading in these environments, a two-dimensional Gaussian distribution for simulating the log- amplitudes of spatiallyadjacent profiles has been proposed and implemented.

\section{INTRODUCTION}

Detailed knowledge of radio propagation inside buildings is essential for successful design of indoor communication systems. Measurement and modeling of the indoor radio propagation channel has been performed and reported by different investigators [1]-[7]. Reference [1] is a comprehensive tutorial-survey coverage of the topic.

The random and complicated indoor radio propagation channel can be characterized using the impulse response approach: at each point in the three-dimensional environment the channel is a linear filter with the complex-valued impulse response

$h(t)=\sum_{k=0}^{N-1} a_{k} \delta\left(t-t_{k}\right) e^{j \theta_{k}}$,

where $\mathrm{N}$ is the number of multipath components, $\left\{a_{k}\right\},\left\{t_{k}\right\},\left\{\theta_{k}\right\}$ are the random amplitude, arrival-time, and phase sequences. respectively, and $\delta$ is the Dirac delta function. The channel is completely characterized by these path variables. This is a wideband model which has the advantage that, because of its generality, it can be used to obtain the response of the channel to the transmission of any transmitted signal by convolving that signal with $h(t)$ and adding noise.

The work reported in this paper is concerned with the analysis and simulation of the signal amplitude sequence $\left(a_{k}\right\}$. Distributions over both local and global areas have been investigated.

\section{MEASUREMENT PLAN AND PROCEDURE}

Using the impulse response approach of Eq. (1), extensive propagation measurements were performed at two office buildings. The purpose of these measurements was full characterization of the two propagation mediums based on elaborate statistical analysis and modeling of the channel's impulse response. A brief description of measurement plan and procedure is provided in this section.

The following measurement plan was executed at each building: four transmitter-receiver antenna separations of 5,10 , 20 , and 30 meters were considered. For each antenna separation 20 locations were visited on the basis of good variation of typical conditions within the building. Each location was carefully selected, with both line-of-sight (LOS) and non-line-of-sight (or obstructed) topographies included. The number of obstructed locations was higher for larger antenna separations, consistent with conditions encountered in real-life indoor portable communication systems. The base (i.e., the fixed) antenna location was also varied throughout the buildings. At each location 75 frequency response profiles at 2 centimeter spacing were measured and recorded. This resulted in a large data base of 6000 frequency response profiles for each building ( 75 profiles per location $\times 20$ locations per antenna separation $\times 4$ antenna separations per building).

The above measurement plan was executed at two dissimilar office buildings. Since geometry of the buildings made it impossible to pick 20 portable locations per fixed site (base), variations in the base site was also permitted for each antenna separation. This procedure brings the added advantage of making the results less biased, as compared to a scenario in which a single base location is selected for all measurements.

The first building measured was NovAtel Communications Ltd. Corporate Office located in Calgary, Alberta, Canada. This is a modern three storey facility containing offices, cubicles, and laboratory space. Measurements were performed on the first and third floors, with both antennas located in the same floor. The second building measured was the Alberta Government Telephone (AGT) Tower also located in Calgary. This building is a high-rise containing primarily offices and cubicles, with some laboratory space. Measurements were performed on the 19 th floor only.

The measurements were performed using a Vector Network Analyzer measuring the frequency response of the indoor propagation channel between two discone antennas. The network analyzer swept frequency band was $900 \mathrm{MHz}$ to $1300 \mathrm{MHz}$ in 500 $\mathrm{KHz}$ steps ( 801 points). The time required was $400 \mathrm{~ms}$ per sweep with 10 sweeps being averaged per measurement, making the actual time for each measurement 4 seconds. All measurements 
were performed at night or on weekends when there were few, if any, other personnel in the vicinity of the measurement setup.

The 12000 frequency response profiles measured using the above procedure were converted to the time domain by classical Fourier analysis. This resulted in an equal number of impulse response profiles. Details of the measurement plan, procedure, and subsequent processing are reported in [2].

\section{SIGNAL AMPLITUDE DISTRIBUTIONS}

\section{Amplitude Fading Distributions}

Major probability distributions encountered in multipath fading environments are Rayleigh, Rice, Nakagami, lognormal, and Suzuki. Rice and Suzuki distributions did not provide good fit to most of the data, and therefore, the results of curve fitting for the other three distributions are reported.

Rayleigh distribution is often mentioned in connection with purely scattering fading models in absence of a strong (LOS) component. It has one parameter and its probability density function (pdf) is given by:

$f(r)=\frac{r}{\sigma^{2}} \exp \left(-\frac{r^{2}}{2 \sigma^{2}}\right) u(r)$

where $r$ is the envelope at a fixed delay and $\sigma$ is the Rayleigh parameter (the most probable value). The mean and variance of this distribution is $\sqrt{ } \pi / 2 \sigma$ and ( $2-\pi / 2) \sigma^{2}$, respectively.

A second distribution often mentioned in connection with fading over large areas were nonstationarities in the channel should be taken into account is the Lognormal distribution with a pdf given by:

$f(r)=\frac{1}{\sqrt{2 \pi} \sigma r} \exp \left\{-(\ln r-\mu)^{2} / 2 \sigma^{2}\right\} u(r)$.

Equation (3) shows that $\ln (r)$ has a normal distribution. This distribution has two parameters $\mu$ and $\sigma$.

A third probability distribution that has been tested successfully to describe envelope fading in multipath environments is the Nakagami or m-distribution with the probability density function

$f(r)=\frac{2 m^{m} r^{2 m-1}}{\Gamma(m) \Omega^{m}} \exp \left\{-\frac{m r^{2}}{\Omega}\right\} U(r)$

where $\Gamma(m)$ is the Gamma function, $\Omega=E\left\{r^{2}\right\}$ and $m=\left\{E\left[r^{2}\right]\right\}^{2}$ $N$ ar $\left[r^{2}\right]$, with the constraint $m \geq 1 / 2$. Nakagami is a general fading distribution that reduces to the Rayleigh for $m=1$ and to the one-sided Gaussian distribution for $m=1 / 2$. It also approximates the Rician and Lognormal distributions.

\section{Local Amplitude Distributions}

Local data refers to the set of 75 profiles collected in one location. To test local amplitude distributions at a fixed delay the Kolmogorov-Smirnov (K-S) goodness of fit test between empirical and theoretical distributions was employed. In the K-S test the maximum "distance" between the theoretical and empirical distributions $D_{N}$ is defined as:
$D_{N}=\max _{x}\left|F(x) \cdot F_{N}(x)\right|$,

where $F(x)$ is the theoretical distribution and $F_{N}(x)$ is the empirical distribution obtained from the ordered sequence of samples [8]. Distribution of $D_{N}$ depends on $N$ (size of the samples), and is independent of the form of $F(x)$. For large $N$ distribution of $N$ is given by:

$\lim _{N \rightarrow \infty} \operatorname{Prob}\left[D_{N}<\frac{C_{N}}{N}\right]=1-2 \sum_{i=1}^{\infty}(-1)^{i-1} \exp \left(-2 i^{2} c^{2}\right)$

This equation can also be written as:

Prob $\left[D_{N}<\varepsilon_{N, 0}\right]=1-\alpha$,

in which $\alpha$ is the significance level and $\varepsilon_{\mathrm{N}, \alpha}$ is the critical value of the test. For large $N$, the threshold value $\varepsilon_{N, \alpha}$ can be written as:

$\varepsilon_{N, \alpha}=\sqrt{-\frac{1}{2 N} \ln \frac{\alpha}{2}}$

To carry out the K-S test $\mathrm{D}_{\mathrm{N}}$ was calculated and compared with $\varepsilon_{N, x}$, which was in turn calculated from the number of samples and the confidence level. If $\mathrm{D}_{\mathrm{N}}$ is smaller than $\varepsilon_{N, \alpha}$, with a confidence level of 1- $\alpha$ the hypothesis $F(x)=F_{N}(x)$ can not be rejected; i.e., $\mathrm{F}(\mathrm{x})$ passes the test.

The empirical distributions were obtained using the moment method. In this method which is relatively easy to implement, theoretical moments of the distributions are obtained and equated with the theoretical moments estimated from the data. Parameters of $F(x)$ are then calculated. Parameter estimation and curve fitting can be performed on a linear scale or on a log scale. For dynamic ranges of data larger than $20 \mathrm{~dB}$, contribution of weak amplitudes for curve fitting on a linear scale is insignificant, and therefore, parameter estimation and curve fitting is performed on a log scale. This corresponds to curve fitting of the empirical data with the normal, log-Rayleigh, and log-Nakagami distributions. To save space mathematical details are not reported here; they can be found in [9] and [10].

Resolution of the experiment was 5 nsec. Prior to data reduction the excess delay axis was divided into small intervals of width 5 nsec. called "bins". Each bin contains either one component or no component. Possibility of more than one component in a bin is excluded. It was observed from the data that probability of receiving components with excess delays larger than $500 \mathrm{nsec}$. is negligible. The excess delay axis was therefore divided into 100 bins. This discrete version of the data was used for the analysis reported in this work.

Each location contains 75 profiles. Therefore, for each bin (i.e., for each excess delay) maximum number of samples is 75. To increase the number of samples data for 5 adjacent bins were combined. This is justified since amplitude of components in adjacent bins have similar statistics. Therefore, curve fitting was performed for portions of the excess clelay axis which are $25 \mathrm{nsec}$. wide and are centered at excess delstys of $25,50,75,100, \ldots \ldots$. $425,450,475 \mathrm{nsec}$. For each portion of the excess delay axis and 
for each theoretical distribution the K-S test was performed with a confidence level of $90 \%$. Fig. 1 shows samples of curve fitting for hoth buildings. Samples of the results are also reproduced in Table I. In this table a "1" indicates that the data passed the K-S test for the corresponding theoretical distribution, and a " 0 " means that it did not pass the test.

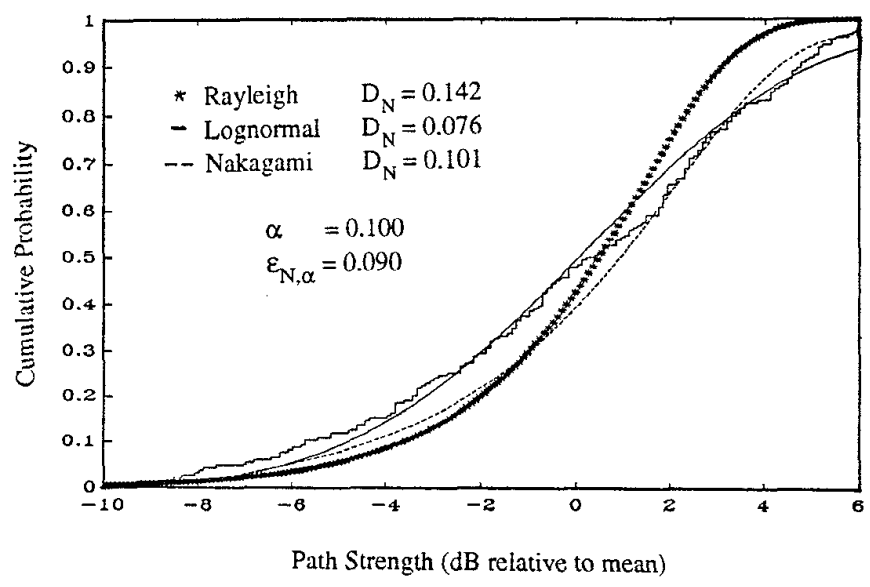

(a)

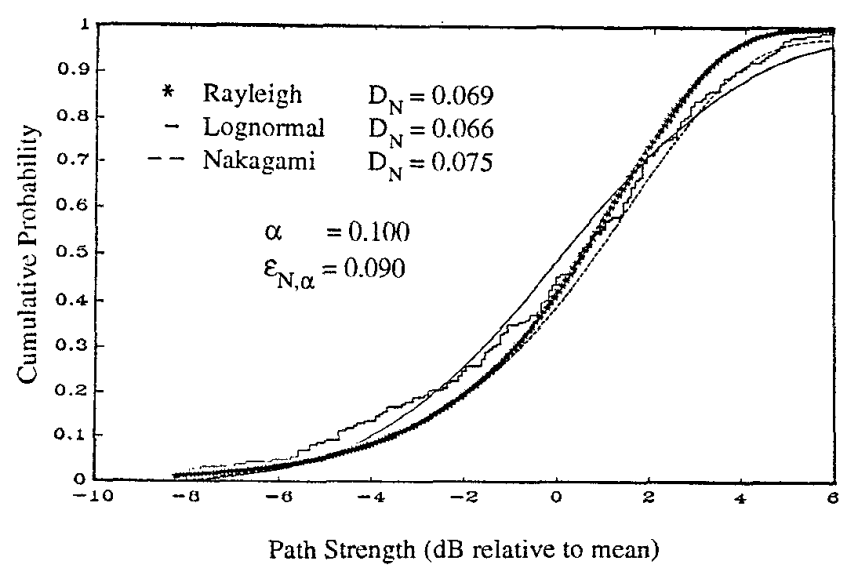

(b)

Fig. 1. Empirical and theoretical local amplitude distributions. (a) Building A, antenna separation of 5 meters, location 1. excess delay of $100 \mathrm{nsec}$ (b) Building B, antenna separation of 30 meters, location 14. excess delay of 25 nsec.

The amount of data analyzed provides statistically significant results. A total of 160 locations for both buildings (20 locations for each one of 4 antenna separations at each one of two buildings) were tested. For each location 19 portions of the excess delay axis were considered. Overall, more than 3000 cases were therefore tested. Results of the local curve fitting (a few of which are reflected in Table I) are summarized in Table II. This table shows that with the confidence level of $90 \%$, in $75.6 \%$ of cases the lognormal distribution passes the Kolmogorov-Smirnov test. These numbers are $36.3 \%$ and $12.6 \%$ for the Nakagami and Rayleigh distributions, respectively. It is concluded, therefore, that lognormal distribution is the best candidate to describe amplitude fading over local areas.
Table I. Local fading distributions, Building A, antenna separation of 5 meters. " 1 " means the distribution passed the K-S test and " 0 " means it did not.

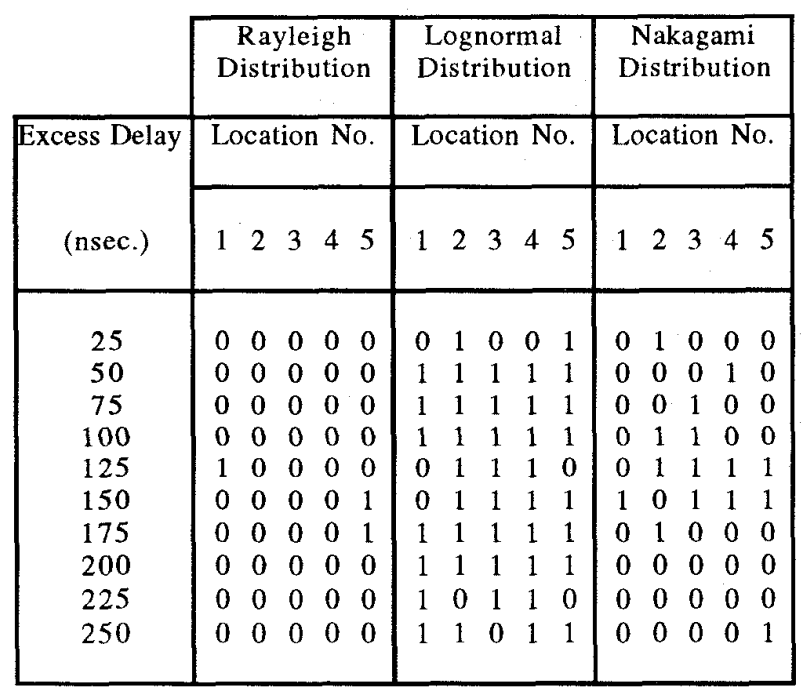

Table II. Percentages of locations for both buildings that passed the K-S test with a confidence level of $90 \%$.

\begin{tabular}{|c|c|c|c|}
\hline $\begin{array}{c}\text { Excess Delay } \\
\text { (nsec.) }\end{array}$ & $\begin{array}{c}\text { Rayleigh } \\
\text { Distribution }\end{array}$ & $\begin{array}{c}\text { Lognormal } \\
\text { Distribution }\end{array}$ & $\begin{array}{c}\text { Nakagami } \\
\text { Distribution }\end{array}$ \\
\hline 25 & 7.5 & 73.3 & 40.0 \\
50 & 11.7 & 79.2 & 44.2 \\
75 & 13.3 & 77.5 & 43.3 \\
100 & 3.3 & 75.8 & 53.3 \\
125 & 10.8 & 80.8 & 40.0 \\
150 & 20.8 & 80.0 & 53.3 \\
175 & 15.0 & 80.8 & 30.0 \\
200 & 15.0 & 80.8 & 24.2 \\
225 & 14.2 & 74.2 & 14.2 \\
250 & 15.0 & 56.7 & 20.0 \\
\hline & & & \\
\hline & 12.6 & 75.9 & 36.3 \\
\hline
\end{tabular}

\section{Global Amplitude Distributions}

Each set of global data consists of 1500 profiles collected at one antenna separation. In the analysis of global data also multipath components for five adjacent bins were combined. This increases the number of samples for each portion of the excess delay axis to several thousands. The theoretical and empirical distributions were obtained using the methods described in the previous section. Mean square error between the two distributions is defined as: 
$M S E=\int_{-\infty}^{\infty}\left[F(x)-F_{N}(x)\right]^{2} d x$

Typical theoretical and empirical global distributions are shown in Fig. 2. The MSE values for Building B, antenna separation of 20 meters are reproduced in Table III. It was observed that for most cases the lognormal distribution provided smaller MSE's as compared to the Nakagami and Rayleigh distributions. For each antenna separation there are 19 portions of excess delay and 19 corresponding MSE values (for each theoretical distribution). The Wilcoxon test on the MSE's was used to determine the best distribution. This test showed that with an average confidence level of $99.04 \%$ the lognormal distribution is better than Nakagami. The test also showed that with an average confidence level of $94.07 \%$ Nakagami is better than Rayleigh. It is therefore concluded that the lognormal distribution is also the hest candidate for describing amplitudes over global areas.

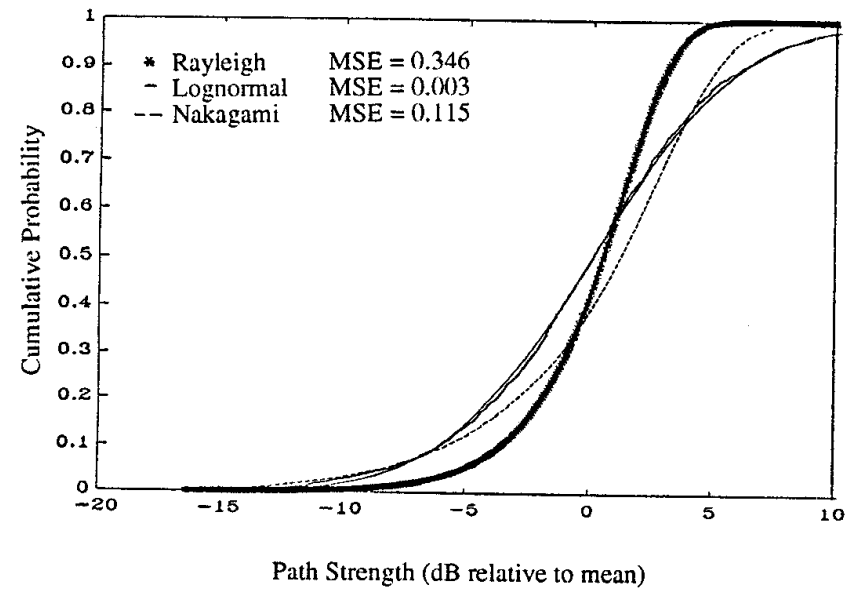

(a)

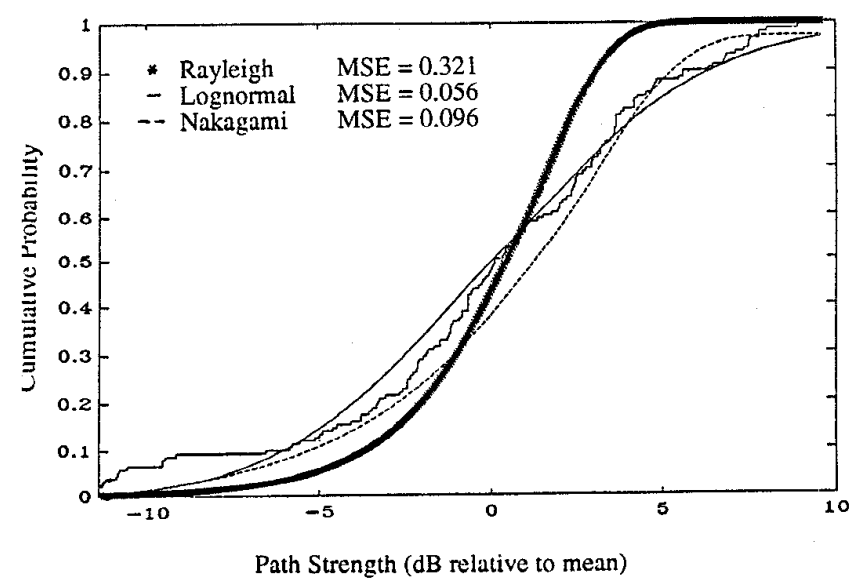

(b)

Fig. 2. Empirical and theoretical global distributions. (a) Building A, antenna separation of 20 meters, excess delay of 100 nsec. (b) Building $B$, antenna separation of 5 meters, excess delay of 375 nsec.
Table III. Mean square error between empirical and theoretical global distributions, Building B, antenna separation of 20 meters.

\begin{tabular}{|c|c|c|c|}
\hline $\begin{array}{c}\text { Excess Delay } \\
\text { (nsec.) }\end{array}$ & $\begin{array}{c}\text { Rayleigh } \\
\text { Distribution }\end{array}$ & $\begin{array}{c}\text { Loglnormal } \\
\text { Distribution }\end{array}$ & $\begin{array}{c}\text { Nakagami } \\
\text { Distribution }\end{array}$ \\
\hline 25 & 0.3176 & 0.0147 & 0.0632 \\
50 & 0.2324 & 0.0147 & 0.0413 \\
75 & 0.3242 & 0.0092 & 0.0755 \\
100 & 0.3133 & 0.0085 & 0.0694 \\
125 & 0.1848 & 0.0133 & 0.0408 \\
150 & 0.2291 & 0.0051 & 0.0666 \\
175 & 0.2603 & 0.0052 & 0.0681 \\
200 & 0.2675 & 0.0039 & 0.0863 \\
225 & 0.1845 & 0.0082 & 0.1138 \\
250 & 0.1495 & 0.0505 & 0.2399 \\
275 & 0.1011 & 0.0474 & 0.1755 \\
300 & 0.1195 & 0.0448 & 0.1370 \\
325 & 0.2589 & 0.0119 & 0.0575 \\
350 & 0.3195 & 0.0085 & 0.0584 \\
375 & 0.2460 & 0.0318 & 0.0930 \\
400 & 0.2701 & 0.0163 & 0.0628 \\
425 & 0.3281 & 0.0442 & 0.1120 \\
450 & 0.3281 & 0.0726 & 0.1726 \\
475 & 0.3824 & 0.0327 & 0.1151 \\
& & & \\
\hline
\end{tabular}

\section{A Proposed Simulation Model}

Theoretical local and global distributions of the signal amplitudes were described in the previous sections. On the basis of these results one can assume that log-amplitude of the multipath components at a fixed excess delay for two impulse response profiles collected at adjacent points in space in the same local environment have a bivariate normal distribution with their correlation coefficient a decreasing function of the sampling distance. The joint density function of two consecutive logamplitudes (to be denoted by $\mathrm{X}_{\mathrm{m}}$ and $\mathrm{X}_{\mathrm{m}-1}$ for simplicity of notation, were the index $m$ numbers the spatially-separated points along a track in the same local area) are therefore given by:

$$
\begin{gathered}
f_{X_{m}, X_{m-1}}\left(x_{m}, X_{m-1}\right)=: \frac{1}{2 \pi \sigma_{m} \sigma_{m-1} \sqrt{1-\rho^{2}}} \exp \left\{-\frac{1}{2\left(1-\rho^{2}\right)}\left[\frac{\left(x_{m}-\mu_{m}\right)^{2}}{\sigma_{m}^{2}}\right.\right. \\
\left.\left.-2 \rho \frac{\left(x_{m}-\mu_{n}\right)\left(x_{m-1}-\mu_{m-1}\right)}{\sigma_{m} \sigma_{m-1}}+\frac{\left(x_{m-1}-\mu_{m-1}\right)^{2}}{\sigma_{m-1}}\right]\right\}
\end{gathered}
$$

where $\mu_{m}=\mathrm{E}\left(\mathrm{X}_{\mathrm{m}}\right), \sigma_{\mathrm{m}}{ }^{2}=\operatorname{Var}\left(\mathrm{X}_{\mathrm{m}}\right), \mu_{\mathrm{m}-1}=\mathrm{E}\left(\mathrm{X}_{\mathrm{m} \cdot \mathrm{l}}\right), \sigma_{\mathrm{m}-1}{ }^{2}=\operatorname{Var}\left(\mathrm{X}_{\mathrm{m}-1}\right)$, and $\rho$ is the correlation coefficient between $\mathrm{X}_{\mathrm{m}}$ and $\mathrm{X}_{\mathrm{m}-1}(1 \mathrm{pl}<1)$. Since $X_{m} \quad(m=1,2, \ldots .$.$) represent log-amplitudes for impulse$ response profiles collected in the same location, they have similar statistics; therefore, $\mu_{\mathrm{m}}=\mu_{\mathrm{m}-1}=\mu$, and $\sigma_{\mathrm{m}}=\sigma_{\mathrm{m}-1}=\sigma$.

A simulation model for generating a consistent set of log-amplitudes is proposed here: 
Step I. A local mean $\mu$ and a local standard deviation $\sigma$ are specified by the user on the basis of the large data base of values obtained throughout the measurements. $\mu$ and $\sigma$ may also be generated according to the appropriate normal distributions, the parameters of which are available from our analysis (but were not reported here due to space limitations).

Step 2. $X_{1}$ for a location is generated according to a $N(\mu, \sigma)$ distribution. $\quad X_{m}, m=2,3, \ldots$. are generated according to a conditional normal distribution, with the condition being the value of $\mathrm{X}_{\mathrm{m}-1}$. This is because if $\mathrm{X}_{\mathrm{m}}$ and $\mathrm{X}_{\mathrm{m}-1}$ are jointly normal with mean $\mu$ and standard deviation $\sigma$, then $X_{m}$ conditioned on $\mathrm{X}_{\mathrm{m}-1}$ has a normal distribution with mean and standard deviations given by:

$\mu_{c}=\mu+\rho\left(x_{m-1}-\mu\right)$

and

$\sigma_{c}=\sigma \sqrt{1-\rho^{2}}$

The correlation coefficient $\rho$ depends on the sampling distance. Numerical values were calculated and reported in [2].

\section{CONCLUSION}

The results of extensive curve fitting for local and global distributions of the amplitude of multipath components for indoor radio propagation data were reported. The data base included 12000 impulse response profiles collected at two modern office buildings. Major result of the analysis is that amplitude fading is lognormal over both local and global areas. On the basis of the results of this work a simulation model for generating amplitude of multipath components at fixed delays for spatially-separated points was proposed. The results of this analysis can be used in the simulation and design of indoor radio communication systems.

The large multipath propagation data base has been further analyzed to obtain full understanding of the channel's timeinvariant impulse response. The corresponding results have been reported in [2]-[4].

\section{ACKNOWLEDGMENTS}

The authors would like to express their gratitude to the Vice President of Research, Sharif University of Technology for funding and supporting this project. Measurements at the two building were carried out by David Tholl and Gerald Morrison of TRLabs, Calgary, Alberta, Canada. Their efforts are also gratefully acknowledged.

\section{REFERENCES}

[1] H. Hashemi, "The indoor radio propagation channel," Proceedings of the IEEE, Vol. 81, no. 7, July 1993.

[2] H. Hashemi, "Impulse response modeling of indoor radio propagation channels." IEEE Journal on Selected Areas in Communications, Special Issue on Wireless Communications, Sept. 1993.
[3] H. Hashemi and D. Tholl, "Statistical modeling and simulation of the RMS delay spread of indoor radic propagation channels," to appear in the IEEE Transaction on Vehicular Technology, 1993.

[4] H. Hashemi and D. Tholl, "Analysis of the RMS delay spread of indoor radio propagation channels," Proceedings of the IEEE International Conference on Communications. ICC'92, Chicago, Illinois, June 14-17, 1992, pp. 875 . 881 .

[5] T.S. Rappaport, S.Y. Seidel, K. Takamizawa, "Statistical channel impulse response models for factory and open plan building radio communication system design," IEEF Transactions on Communications, Vol. 39, No. 5, pp 794-807, May 1991.

[6] D. M. J. Devasirvatham, "Time delay spread and signal level measurements of $850 \mathrm{MHz}$ radio waves in building environments," IEEE Trans. on Antennas and Propagation, Vol. AP-34, No. 11, pp. 1300-1305, Nov. 1986.

[7] R. J. C. Bultitude, S. A. Mahmoud, W. A. Sullivan, "A comparison of indoor radio propagation characteristics a $910 \mathrm{MHz}$ and $1.75 \mathrm{GHz}$," IEEE Journal on Select. Areas in Comm., Vol. 7, No. 1, pp. 20-30, Jan. 1989

[8] Z. W. Birnbaum, "Numerical tabulation of the distribution of Kolmogorov's statistics for finite sample size," American Statistical Association Journal, pp. 425-441, Sept. 1952.

[9] W. L. Shepherd and P. Milnarich Jr., "Basic relations between a Rayleigh distributed randomly varying voltage and a decibel record of voltage," Proceedings of the IEEE, pp. 1765-1766, Dec. 1973.

[10] W. C. Hoffman, "Some statistical methods of potentia] value in radiowave propagation investigations," from Statistical Methods in Radiowave Propagation, Pergamon Press, 1960.

Homayoun Nikookar was born on March 1, 1962. He received the B.S. and M.S. degrees both in Electrical Engineering from Sharif University of Technology, Teheran, Iran, in 1985 and 1986 , respectively. He is currently pursuing the $\mathrm{PhD}$ degree, also in Electrical Engineering at Amirkabir University of Technology. Since 1986 he has been a lecturer at both Sharif University of Technology and Amirkabir University of Technology. His research interests include mobile and indoor radio communications. Mr. Nikookar is a member of IEEE.

Homayoun Hashemi is associate professor of electrical engineering at Sharif University of Technology, in Teheran, Iran. He received the M.S. and PhD degrees in electrical engineering from the University of California at Berkeley. In the past he was a member of technical staff at Bell Telephone Laboratories in Holmdel, New Jersey, served as a consultant to Stanford Research Institute in Menlo Park, Calif., and was a visiting scholar at the Electrical Engineering Department of the University of Ottawa, and at TRLabs in Calgary, Alberta, Canada. He spent a one-year sabbatical leave at NovAtel Communications Ltd. in Calgary. He has also served as a consultant to the Iranian Telecommunications Research Center and the Telecommunications Company of Iran. 